CMAJ Public Health Fact Sheet

\section{Influenza and the influenza vaccine}

\section{What is influenza and what are the symptoms?}

Influenza, or the "flu," is an infection caused by the influenza virus. The virus infects the nose and throat, and it can sometimes spread to the lungs. Influenza commonly causes acute fever, cough, chills, tiredness, body aches and, in young children, ear aches. People with influenza are often too sick to go to work, school or daycare. Unlike the viruses that cause the common cold, the influenza virus can cause severe illness like pneumonia, especially in people who are very young or very old, or who have conditions such as cancer, heart disease, asthma and diabetes. In Canada, more than 4000 people die from influenza each year.

\section{When is "flu season" in Canada?}

Influenza comes every year during the winter months (January-March). When influenza arrives in a community, there is a sharp rise in the number of doctor visits, admissions to hospital and respiratory outbreaks in long-term care facilities. Many children miss school and many people miss work because of influenza infections. The specific types of influenza viruses change a little each year.

\section{How is influenza spread?}

Infected people spread influenza by coughing or sneezing, or by touching things with their hands after coughing or sneezing. Coughing and sneezing spreads the influenza virus through small droplets that travel up to a metre ( 3 feet) and land on nearby people or objects. To help stop the spread of influenza, you should "cover your cough." Cover your mouth and nose with a tissue or your sleeve - do not cough into your hands. Put the used tissue into a wastebasket, and clean your hands with soap and water or an alcohol-based hand sanitizer. Adults with influenza can spread the virus from I day before their symptoms ap- pear to up to 7 days after their symptoms appear. Children may spread the infection for more than 7 days, especially if their symptoms persist.

\section{How can influenza infection be prevented?}

There are only a few ways to decrease the chance of getting influenza. The combination of receiving the influenza vaccine and cleaning your hands many times a day during flu season can prevent most influenza cases. During an influenza outbreak, anti-influenza drugs, like oseltamivir, are sometimes used to prevent the spread of influenza to patients at high risk (e.g., those in a long-term care facility).

\section{What is the influenza vaccine?}

The "flu shot" is made of inactive (killed) influenza virus particles. The flu shot does NOT cause influenza because there is no live virus in the vaccine. The influenza virus used in the flu shot is grown in eggs, so anyone who is allergic to eggs cannot get a flu shot.

\section{Why do I have to get a flu shot every year? How long does the flu shot protect me?}

Because the influenza virus changes every year, a new vaccine has to be developed each year. The flu shot protects for less than I year, and it protects only against the types of influenza virus in the vaccine. For the best protection, the flu shot should be taken every year.

\section{Does the flu shot prevent influenza?}

How well the flu vaccine works depends on how closely the virus in the vaccine matches the type of influenza virus in the community. If there is a good match, then the vaccine prevents illness in $70 \%$ to $90 \%$ of people. Even when the match is not so good, the flu shot still decreases the rate of serious illness. The flu shot does not work if it is given after the symptoms have started. For best results, the flu shot should be given before influenza comes into the community.

\section{What are the side effects of the flu shot?}

Most people do not have any side effects other than a sore arm at the injection site for I or 2 days. Occasionally, a mild fever or muscle aches and pains may occur - but this is not the flu. These signs suggest that you are developing a good defence against the types of flu in the vaccine. Serious side effects, like hives, are very rare unless the person is allergic to eggs. Development of Guillain-Barré Syndrome, a nerve disorder, following flu vaccination is very rare (about I in I 000 ooo people vaccinated).

\section{Who should be vaccinated?}

People at risk of serious complications because of influenza and the people who care for them (e.g, in the health care system or at home) are strongly urged to get the flu shot. This includes people aged 65 years or older, those aged 6-23 months, those with select chronic health conditions (e.g., heart disease, asthma, cystic fibrosis, diabetes, cancer, renal disease and anemia), people of any age who live in a long-term care facility and pregnant women. People who provide care to people with the health conditions listed above should also get the flu shot to prevent the spread of influenza. Healthy people between the ages of 2 and 64 years also benefit from receiving the vaccine.

Noni MacDonald MD MSc

Section Editor, Public Health, CMAJ

Erica Weir MD MSc

Associate Editor, CMAJ

Joanne M. Langley MD

Division of Infectious Diseases

Department of Pediatrics

Dalhousie University,

Halifax, NS

Competing interests: None declared.

Une version française de cet article est disponible à l'adresse www.cmaj.ca/cgi /content/full/I77/9/IO28/DCI 